EPJ Web of Conferences 49, 07004 (2013)

DOI: $10.1051 /$ epjconf/20134907004

(C) Owned by the authors, published by EDP Sciences, 2013

\title{
Asymmetries in production of the top quark
}

\author{
Regina Demina ${ }^{1, a}$ \\ ${ }^{1}$ University of Rochester, Rochester, Ny, 14627
}

\begin{abstract}
I give an overview of the experimental results on the top quark production asymmetries from the Tevatron and LHC. Particular attention is given to the discussion of the comparison of the results from these two machines.
\end{abstract}

\section{Introduction}

Forward-backward asymmetry in the production of topantitop pairs in proton-antiproton collisions has been reported by the D0 [1] and CDF [2] collaborations since 2008. With the increased statistics, the significance of the deviation between the Standard Model prediction and the experimental results keeps increasing [3], [4]. Since the effect was first reported the precision and sophistication of the theoretical prediction has greatly increased [5], including next-to-leading logarithms [6], yet stopping short of the full NNLO calculation [7]. Improved calculations within the framework of QCD and those incorporating the electroweak effects [8] point towards higher values of the asymmetry than originally predicted [9], yet still smaller than the experimentally measured values. Meanwhile, a number of beyond the Standard Model scenarios were suggested to explain the effect [10], most of them coming into some level of contradiction with the other observations from the LHC and Tevatron, most notably the absence of the like-sign top pair production [11] and resonances in the dijet spectrum [12]. The charge asymmetry in top pair production at the LHC reported by the CMS [13] and Atlas [14] collaborations is in agreement with the Standard Model prediction. In these proceedings I review the present status of the Tevatron measurements and discuss the consistency of these results with the LHC observations.

\section{Tevatron results}

\subsection{Definitions}

We refer to the event, where the top (anti) quark is moving in the direction of (anti)proton as "forward", otherwise it is called "backward". The difference between the number of forward and backward events divided by their sum is called forward-backward $t \bar{t}$ asymmetry $A_{F B}$. The sign of the top quark is determined by the sign of lepton from $W$ boson decay. This implies that the $t \bar{t}$ system must be fully

\footnotetext{
ae-mail: regina@pas.rochester.edu
}

Table 1. Summary of the Tevatron measurements of fully reconstructed asymmetry $A_{F B}$ and lepton-based asymmetry $A_{F B}^{l}$

\begin{tabular}{lllll}
\hline Quantity & Channel & Experiment & Value, \% & ref \\
\hline$A_{F B}$ & l+ jets & CDF & $16.4 \pm 4.5$ & {$[4]$} \\
$A_{F B}$ & l+ jets & D0 & $19.6 \pm 6.4$ & {$[3]$} \\
$A_{F B}$ & dilepton & CDF & $42 \pm 16$ & {$[16]$} \\
$A_{F B}$ & & theory & $6.6 \pm 2.0$ & {$[17]$} \\
\hline$A_{F B}^{l}$ & l+ jets & CDF & $6.5 \pm 2.0$ & {$[4]$} \\
$A_{F B}^{l}$ & $l+$ jets & D0 & $15.2 \pm 3.9$ & {$[4]$} \\
$A_{F B}^{l}$ & dilepton & D0 & $5.8 \pm 5.3$ & {$[18]$} \\
$A_{F B}^{l}$ & & theory & $2.1 \pm 0.1$ & {$[19]$} \\
\hline
\end{tabular}

reconstructed, using kinematic fitting techniques. The reconstructed $t \bar{t}$ asymmetry is "diluted" because of the detector resolution effects and due to a possibility of wrong assignments of the decay objects to the primary parton. To infer the asymmetry at production from the reconstructed asymmetry unfolding techniques are employed. The numbers quoted in this article for production level asymmetries.

A simpler observable is asymmetry of lepton from top quark decay. Lepton charge and direction are usually measured with excellent precision, thus unfolding simply involves a correction for acceptance. Asymmetry based on the lepton direction $A_{F B}^{l}$ is defined as the difference between the number of events, where positive (negative) lepton follows the direction of (anti)proton and the number of events where lepton goes into another hemisphere, divided by the total number of events. Given the asymmetry in top production based on any theoretical framework, it is straightforward to predict the value of $A_{F B}^{l}$. It provides a valuable cross check to a fully reconstructed asymmetry. Recent theoretical developments have shown that it also provides additional information to help separate between different models [15].

\subsection{Summary of the results}

In figure 1 we present the distribution in the difference in rapidity of top and anti top quarks $\Delta y$, which is the ba- 
Table 2. Summary of the LHC measurements of top production charge asymmetry

\begin{tabular}{lllll}
\hline Quantity & Channel & Experiment & Value, \% & ref \\
\hline$A_{C}$ & $l+$ jets & CMS & $0.4 \pm 1.5$ & {$[13]$} \\
$A_{C}$ & $l+$ jets & Atlas & $-1.8 \pm 3.6$ & {$[14]$} \\
$A_{C}$ & dileptons & Atlas & $5.7 \pm 2.8$ & {$[21]$} \\
\hline
\end{tabular}

sis for measuring the fully reconstructed asymmetry $A_{F B}$, as observed by the D0 and CDF experiments. There is a shift towards the positive values of $\Delta y$ compared to expectation. Table 1 summarizes the Tevatron results on $A_{F B}$ and $A_{F B}^{l}$ measured in the channel, where one of the $W$ bosons from top decay leptonically, referred to as $l+$ jets channel, and when both $W$-bosons decay leptonically, referred to as dileptons. The results are not all based on the analysis of the full statistics, so some improvement in the significance of the discrepancy between the theoretical prediction and the observation is expected to come from the Tevatron. Yet, ultimately, it will be up to the LHC experiments with their high statistics in $t \bar{t}$ samples to resolve this issue. In the next section I comment on the caveats of comparing the LHC and the Tevatron results.

\section{LHC results}

\subsection{Definitions}

Since the colliding particles in the LHC are charge symmetric, the definition of the forward backward asymmetry becomes meaningless. At the same time, valence quarks, on average, have a higher momentum than sea antiquarks. Thus, the direction of the incoming quark can be inferred from the boost of the $t \bar{t}$ system. Top preference towards the incoming quark direction would result in top quarks produced with higher absolute rapidity $\left(\left|y_{t}\right|\right)$ compared to that of anti tops $\left(\left|y_{\bar{t}}\right|\right)$. At the LHC we define a charge asymmetry $A_{C}$ as the difference between the number of events, where the difference in absolute rapidity between top and anti top is positive, and the ones, where this difference is negative, divided by the total number of events.

\subsection{Production mechanisms and associated asymmetries}

The dominant production mechanism of top-antitop pairs at the Tevatron is due to quark-antiquark annihilation $q \bar{q} \rightarrow t \bar{t}$. The asymmetry measured at the Tevatron is inherently associated with this production mechanism, whether top production is solely due to the Standard Model processes, or some new physics mediates top pair production in $s$ or $t$ channels. At the LHC, on the other hand, top pairs are produced predominantly via gluon fusion, which is inherently symmetric. Quark-qantiquark annihilation is responsible only for less than $10 \%$ on the top pair production. Quark-gluon fusion $q g \rightarrow t \bar{t} q$ contributes a non-negligible fraction especially in the central region. Figure 2(left) shows the relative contributions of different production mechanisms as a function of the absolute value of the sum of rapidities of top and anti top, as simulated by POWHEG [20]. Quark-antiquark annihilation becomes more prominent in the region of high rapidities, while the central region is dominated by $g g$ and $q g$ production. The plot on the right presents the distribution in the difference in absolute rapidities of top and anti top for these different production mechanisms. The asymmetry in this distribution is the basis for determining the charge asymmetry $A_{C}$. It is important to note that, while gluon fusion is symmetries around zero, the other processes are not. The charge asymmetry in top events produced in $q \bar{q}$ annihilation is $2.1 \%$, while $q g$ corresponds to $1.7 \%$ charge asymmetry. The fact that $q g$ production contributes to the asymmetry at LHC, while it is not important for the Tevatron measurement, adds to the complexity of the comparison between the two colliders.

\subsection{Summary of the results}

CMS and Atlas measurements of the charge asymmetry in top pair production are summarized in table 2. All these results are consistent with the Standard Model prediction, yet the question of consistency with the Tevatron results can only be addressed in the framework of a specific model. Figure 3 presents the forwards-backward asymmetry from the Tevatron vs charge asymmetry from the LHC with the results from CDF, D0, CMS and Atlas plotted as bands. Predictions from the Standard Model as well as from the parameter scan of several beyond the Standard Model scenarios are overlaid [22]. It is clear that, while the LHC results started excluding some models, there is a number of them that are consistent with the results from both colliders. 

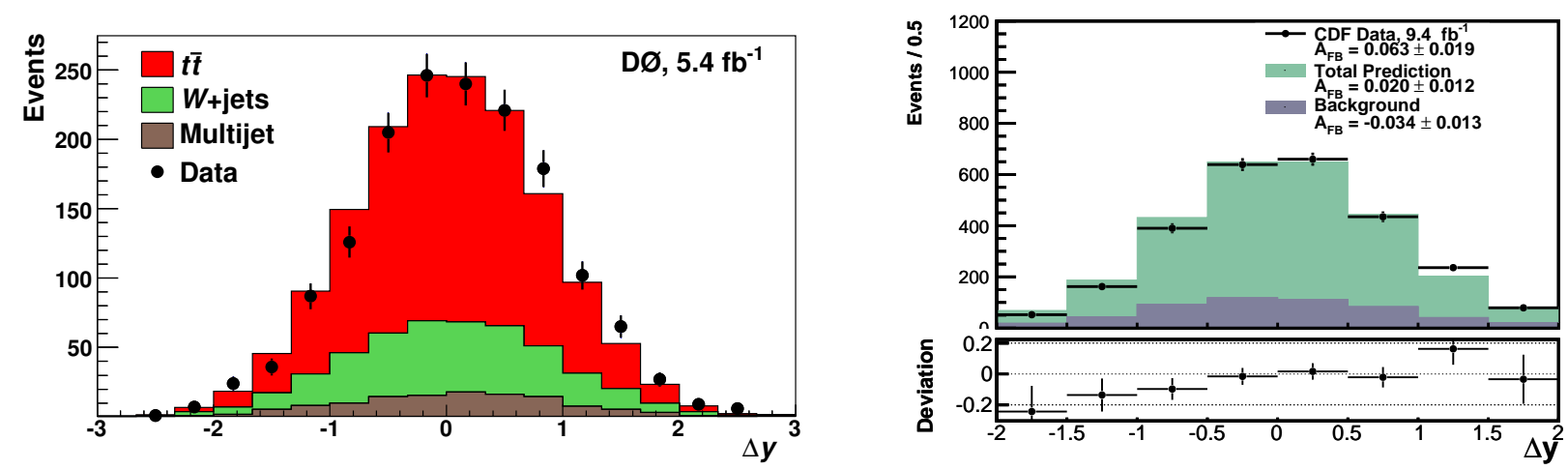

Figure 1. Distributions in $\Delta y_{t}$ in fully reconstructed $t \bar{t}$ events recorded by D0 (left) and CDF (right) detectors at Tevatron.
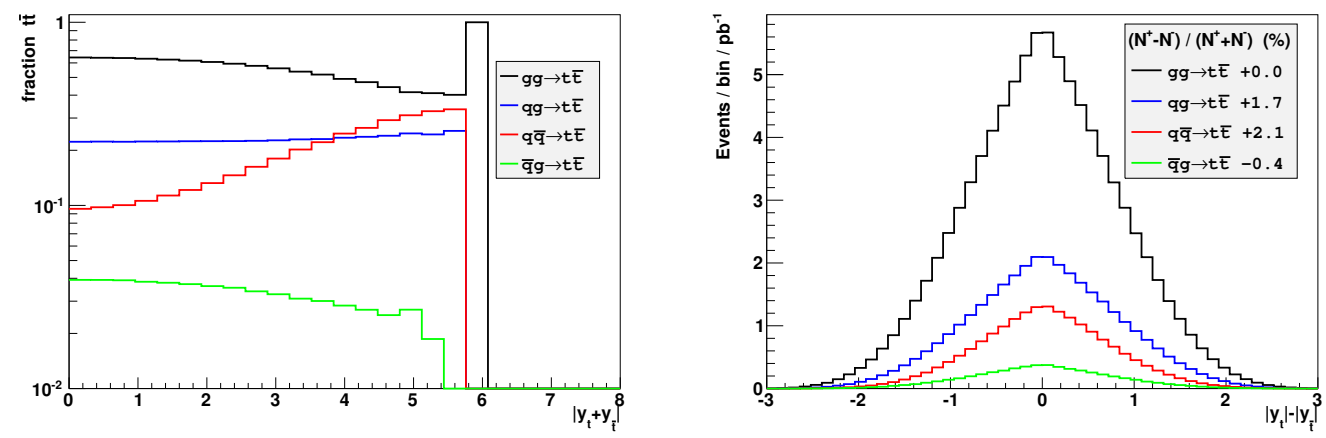

Figure 2. Fractions of different mechanisms responsible for $t \bar{t}$ production in LHC as a function of sum of rapidities of top and anti top quarks (left). Difference in absolute values of rapidities of top and anti top quarks (the basis for measuring the asymmetry at LHC) for different production mechanisms (right).

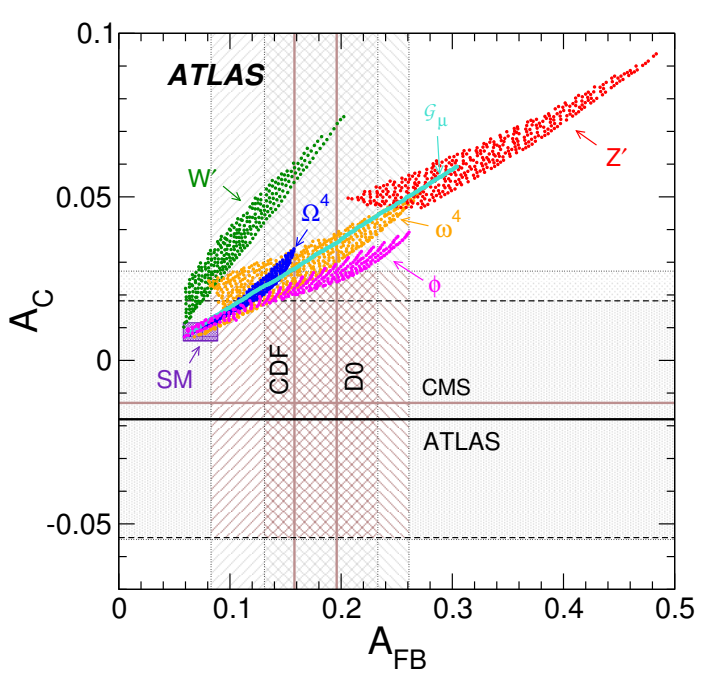

Figure 3. Charge asymmetry in top production at LHC vs forward-backward asymmetry at Tevatron: measurements compared to predictions of a variety of beyond the Standard Model models.

\section{Section title}

\section{References}

[1] V. M. Abazov et al (D0 Collaboration), Phys. Rev. Lett. 100, 142002 (2008).
[2] T. Aaltonen et al (CDF Collaboration), Phys. Rev. Lett. 101, 202001 (2008).

[3] V. M. Abazov et al (D0 Collaboration), Phys. Rev. D 84, 112005 (2011).

[4] T. Aaltonen et al (CDF Collaboration), Phys. Rev. D 83, 112003 (2011).

[5] P. Skands, B. Webber, and J. Winter, J. High Energy Phys. 07 (2012) 151; K. Melnikov, A. Scharf, and M. Schulze, Phys. Rev. D 85, 054002 (2012); S. Dittmaier, P. Uwer, and S. Weinzierl, Eur. Phys. J. C 59, 625 (2009).

[6] P. Baernreuther, M. Czakon, A. Mitov, Phys.Rev.Lett. 109 132001(2012)

[7] M. Czakon, A. Mitov JHEP 1212 054(2012)

[8] W. Hollik and D. Pagani, arXiv:1107.2606 [hep-ph] (2011).

[9] J. H. Kuhn and G. Rodrigo, Phys. Rev. Lett. 81, 49 (1998).

[10] Some recent reviews: M. Gresham, I.-W. Kim, and K. Zurek, Phys. Rev. D 84, 034025 (2011); Q.-H. Cao, D. McKeen, J. L. Rosner, G. Shaughnessy, and C. Wagner, Phys. Rev. D 81, 114004 (2010).

[11] G. Aad et al (Atlas Collaboration), JHEP 1204 (2012) 069; S. Chatrchyan et al (CMS Collaboration), JHEP 1108 (2011) 005. 
[12] S. Chatrchyan et al (CMS Collaboration), Phys.Lett. B704 (2011) 123-142; G. Aad et al (Atlas Collaboration), JHEP 1301 (2013) 029.

[13] S. Chatrchyan et al (CMS Collaboration), Phys.Lett. B 717 129-150 (2012).

[14] G. Aad et al (Atlas Collaboration), Eur.Phys.J. C72 2039 (2012).

[15] A. Falkowski, M. Mangano, A. Martin, G. Perez, J. Winter. CERN-PH-TH-2012-35.

[16] http://www-cdf.fnal.gov/physics/new/top/2011/DilAfb/

[17] Based on MCFM simulation: J. M. Campbell and R.

K. Ellis, Phys. Rev. D 60, 113006 (1999).
[18] V. M. Abazov et al (D0 Collaboration), Phys. Rev. D 87, 011103(R) (2013)

[19] S. Frixione and B. R. Webber, J. High Energy Phys. 06, 029 (2002);

S. Frixione et al, J. High Energy Phys. 08, 007 (2003).

[20] C. Oleari, Nucl.Phys.Proc.Suppl. 205-206 (2010) 3641.

[21] ATLAS Collaboration. Jun 4, 2012, ATLAS-CONF2012-057.

[22] J. Aguilar-Saavedra and M. Perez-Victoria, Phys.Rev. D84 115013(2011). J. Aguilar-Saavedra and M. Perez-Victoria, JHEP 1109097 (2011). 